Ann. Biol. anim. Bioch. Biophys., I974, 14 (4-B), 769-780.

\title{
APPARITION, ORIGINE ET ÉVOLUTION DES TISSUS ADIPEUX ÉPIDIDYMAIRE ET PÉRICARDIAQUE DU FETUS DE PORC
}

\author{
Françoise DESNOYERS et N. VODOVAR \\ avec la collaboration technique de S. Delpal \\ Station de Recherches de Nutrition, \\ Centre national de Recherches zootechniques, I. N.R. A., \\ 78350 Jouy en Josas

\section{RÉSUMÉ}

Chez des fœtus de Porc, dont l'âge varie entre 60 jours et I 3 jours veille du jour présumé de la naissance, le moment d'apparition, l'origine et l'évolution des tissus adipeux péricardiaque et épididymaire ont été étudiés. Il a été montré que, dans nos conditions d'expérience, l'apparition des premiers éléments des tissus adipeux péricardiaque et épididymaire a lieu aux environs du $7 \mathrm{o}^{\mathbf{e}}$ jour de la gestation.

L'étude ultrastructurale des cellules sans lipides, y compris les péricytes, et des premières cellules avec des inclusions lipidiques présentes à l'emplacement des tissus adipeux péricardiaque et épididymaire nous a permis de conclure à l'origine périvasculaire de ces deux tissus adipeux.

Il a été constaté que l'apparition des cellules endothéliales libres, les préadipocytes, est un processus continu durant la vie foetale et que l'intensité de la prolifération de ces futurs adipocytes est étroitement liée à la formation des nouveaux capillaires sanguins.

En tenant compte de l'aspect ultrastructural des cellules, au stade de l'apparition des premières inclusions lipidiques, et de leur évolution, nous pouvons confirmer qu'à tout moment ces cellules ont l'aspect de cellules adipeuses à graisse blanche.

Les résultats obtenus sont discutés et en l'absence de travaux sur les mêmes tissus adipeux, ils sont comparés avec les renseignements existant pour les tissus adipeux des autres emplacements.

\section{IN'TRODUC'TION}

De longue date et encore plus ces dernières années, les tissus adipeux, sous différents aspects et par des techniques différentes, ont été l'objet de multiples investigations. Cependant nos connaissances, en particulier morphologiques, ont souvent été considérées comme insuffisantes (WELLS, I940; BARRNETT, I962; ShELDON, I965) et il faut convenir que dans ce domaine, un nombre important de 
problèmes restent incomplètenment étudiés ou mal connus. L'origine même de ce tissu qui a donné lietı à de longues controverses entre chercheurs (TOLDT, I870; Flemming, I871 ; WASSermann, I926; NApolitano et FaWcetT, I958; Simon, I962 ; Vodovar, Desnoyers et François, I97 I Desnoyers, I973) n'a été étudiée, même en microscopie photonique, que pour un nombre réduit d'espèces et pour quelques emplacements seulement des tissus adipeux. Cela est dû au fait que pendant longtemps et encore à l'heure actuelle, pour une large part de l'opinion, il n'est pas fait de distinctions morphologiques, pourtant bien réelles, entre les tissus adipeux suivant leurs emplacements et suivant les espèces. D'autre part, le moment d'apparition des tissus adipeux suivant les espèces, leur développement au stade fœetal puis le mode de prolifération et de renouvellement des cellules adipeuses n'ont été que peu ou pas étudiés.

Il nous paraît cependant que la connaissance de ces problèmes est une voie d'approche nécessaire à l'étude des facteurs impliqués dans le développement des tissus adipeux permettant, par la suite, de contrôler et d'orienter l'évolution de la masse adipeuse normale et pathologique tant dans un but sanitaire que pour des raisons économiques.

Dans cette optique nous avons, depuis plusieurs années, entrepris des études morphologiques systématiques des tissus adipeux des différents emplacements chez le Porc et chez le Rat (Desnoyers, I973).

Les présentes observations concernent le moment d'apparition, l'origine et l'évolution des tissus adipeux épididymaire et péricardique chez le fœtus de Porc. Ces deux tissus sont étudiés en même temps et comparativement en raison de leur apparition précoce et simultanée bien que leur évolution ne soit pas toujours identique.

La nomenclature que nous utilisons est celle définie précédemment (VoDOVAR, DESNOYERS et FrançOIS, I97I).

Les observations ont été faites en microscopie photonique sur des coupes semifines pour rendre compte de l'aspect topographique de la distribution des différents éléments des tissus adipeux alors que la cytologie a été effectuée en microscopie électronique, seule capable de nous montrer les caractéristiques ultrastructurales des éléments cellulaires et leur évolution.

\section{MATÉRIEL, E'T MÉTHODES}

Des observations préliminaires sur le foetus de Porc nous ont montré que, chez cette espèce, l'apparition des premières cellules adipeuses est toujours postérieure au $60^{\mathrm{e}}$ jour de la gestation et cela quel que soit l'emplacement des tissus.

Les fotus de Porc ont été prélevés sur des truies de race Large white recevant un régime équilibré. Entre le $60^{\mathrm{e}}$ et le I I $3^{\mathrm{e}}$ jour de la gestation, veille du jour présumé de la naissance, les prélèvements ont été fait soit tous les deux jours, soit tous les jours pendant la période d'apparition des premières inclusions lipidiques. Après avoir anesthésié les truies à jeun, une incision est pratiquée au-dessous de l'ombilic. L'utérus et le placenta sont alors ouverts et l'extirpation du fœetus a lieu, ainsi que la résection de la portion d'utérus lui correspondant, ce qui permet de conserver le reste de la portée.

Les fotus mâles ainsi obtenus sont rapidement anesthésiés et des prélèvements sont faits au niveau de l'emplacement prédéterminé du tissu adipeux épididymaire lorsque ces tissus ne sont pas observables macroscopiquement ou à la loupe et par la suite en différents points de ces tissus. Pour le tissu péricardiaque, nous avons généralement prélevé le péricarde fibreux en totalité et nous l'avons immédiatement immergé dans le fixateur. 
La fixation avec le tétroxyde d'osmium à 2 p. Ioo dans le tampon phosphate à o, I M selon Millonig (I96I) a été pratiquée in situ chaque fois que cela a été possible pendant 2 à 5 minutes puis, après extirpation des tissus, pendant un temps variant de 2 à $24 \mathrm{~h}$ à $4^{\circ} \mathrm{C}$. Une fixation prolongée, comme cela a déjà été indiqué (Desnoyers, 1973) est faite dans le but d'obtenir une meilleure préservation des lipides.

Après plusieurs lavages dans le tampon phosphate, les tissus ont été rapidement déshydratés dans des bains d'acétone de titre croissant et l'inclusion a été faite dans la "mixture " d'Epon 812 selon LUfT (I96r). Les blocs ont été coupés à l'ultratome LKB III avec un couteau de verre ou de diamant. Les coupes, après avoir été contrastées sur grille par l'acétate d'uranyle à o,5 p. I00 dans l'alcool à $50^{\circ}$ puis, après séchage, par le citrate de plomb selon Reynolds (I963), ont été observées au Siemens Elmiskop I A.

Pour pouvoir étudier simultanément et sur le même bloc la répartition des éléments du tissu adipeux, des coupes semi-fines de I ou $2 \mu m$ ont été préparées soit avec l'ultratome LKB III, soit avec un Pyramitome LKB. Les coupes ont alors été colorées au bleu de méthylène et azur selon LEESON et LEEson (I970) et observées sur un microscope Leitz Orthoplan.

\section{OBSERVATIONS}

\section{I. - Apparition et origine des tissus adipeux péricardiaque et épididymaire}

a) Moment d'apparition des tissus adipeux péricardiaque et épididymaire.

Le moment d'apparition d'un tissu adipeux à un emplacement déterminé est caractérisé par la présence, à ce niveau, des premières cellules avec des inclusions lipidiques.

Sur les foetus prélevés avant et au $69^{\mathrm{e}}$ jour de la gestation, c'est-à-dire 45 jours avant la naissance, il n'a pas été observé de cellules avec des inclusions lipidiques, ni au niveau du péricarde fibreux ni à l'emplacement du tissu adipeux épididymaire. A cet âge, seules les cellules pouvant être considérées comme de futurs adipocytes sont présentes (fig. I).

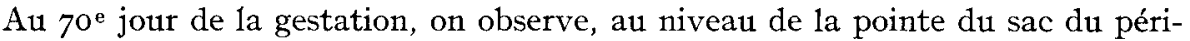
carde fibreux chez tous les fotus étudiés et chez presque tous au niveau du tissu adipeux épididymaire un nombre réduit de cellules avec des inclusions lipidiques de taille et de forme variables (fig. 2).

A partir du $72^{\mathrm{e}}$ jour de la gestation, la présence de cellules avec des inclusions lipidiques devient constante à ces deux emplacements et leur nombre croît progressivement au fur et à mesure que la date présumée de la naissance approche.

On peut donc conclure que pour ces fœtus, dont les mères reçoivent un régime équilibré, 1'apparition des premiers éléments des tissus adipeux péricardiaque et épididymaire se fait aux environs du $7 \mathrm{o}^{\mathrm{e}}$ jour de la gestation.

b) Origine des préadipocytes péricardiaques et épididymaires.

L'étude ultrastructurale des cellules avec des inclusions lipidiques des deux emplacements, prélevés sur des fœtus âgés de 70 jours et des cellules sans lipides, prélevées $\mathrm{I} 2 \mathrm{~h}$ auparavant à des niveaux correspondants, permet de faire un certain nombre de constatations mettant en évidence l'origine des cellules adipeuses de ces deux tissus.

Sur les coupes de péricarde fibreux et de tissu épididymaire de fœetus de Porc âgés de 69 jours, on observe des capillaires sanguins qui, à cet âge, sont peu nombreux et en voie de formation (fig. 3 et 4 ). Ces capillaires, qui ont une paroi de struc- 
ture continue formée de cellules endothéliales jointives, sont entourés par une lame basale plus ou moins apparente (fig. 3 et 4 ). On trouve autour de chaque capillaire un nombre variable de cellules dont certaines sont encore en contact avec les cellules endothéliales. Ces dernières sont entourées par une lame basale commune à celle du capillaire, ce qui indique qu'il s'agit de péricytes (fig. 4). Dans les tissus adipeux péricardiaque et épididymaire, les cellules libres disséminées autour des capillaires sont entourées elles aussi par une lame basale cependant parfois peu apparente. La forme de ces cellules, généralement allongée, varie en fonction de l'incidence de la coupe. Le noyau occupe le plus souvent une position centrale, il est de forme ovale avec un nucléoplasme inégalement contrasté et possède deux ou trois nucléoles bien apparents. L,es éléments cellulaires sont, à cet âge du fœtus, incomplètement différenciés (fig. 3 et 4 ) : la matrice fondamentale du cytoplasme est très peu contrastée, les mitochondries de petite taille ont une matrice très claire, des crêtes peu nombreuses et sans orientation, l'appareil de Golgi est peu développé et le reticulum endoplasmique se réduit à quelques vésicules de reticulum lisse (RE,L) et à de rares tubules de reticulum rugueux (RER).

Ia population des cellules libres du tissu adipeux épididymaire est plus polymorphe que celle du tissu adipeux péricardiaque. On trouve à côté d'une large majorité de cellules dont l'ultrastructure est tout à fait comparable à ce qui a été décrit précédémment, une faible fraction de cellules ayant un aspect comparable à celui des fibroblastes. On trouve également des fibres conjonctives qui n'ont pas été notées au niveau du tissu adipeux péricardiaque.

Sur les coupes de péricarde fibreux et de tissu épididymaire de foetus de Porc âgé de 70 jours, on observe les mêmes éléments que précédemment, plus quelques cellules contenant des inclusions lipidiques qui sont toujours plus éloignées des capillaires sanguins que les cellules sans lipides dont l'ultrastructure est tout à fait comparable à ce qui a été décrit pour le fotus de 69 jours. Les cellules avec des inclusions lipidiques également entourées par une lame basale, ont toujours, en raison des lipides accumulés, un volume plus important (fig. 2) que celui des cellules sans lipides. Leur forme est généralement plus ramassée que celle des cellules sans lipides des fœtus de 69 jours (fig. I). Leur noyau encore en position centrale, tend vers une forme plus sphérique. Parmi les éléments cellulaires, l'aspect de certaines mitochondries diffère de celui des cellules sans lipides : la matrice est plus contrastée et les crêtes sont plus nombreuses (fig. 2).

La répartition des inclusions lipidiques, variable en taille et en nombre, dans les cellules adipeuses péricardiaques et épididymaires du fotus de Porc de 7o jours, se fait apparemment au hasard dans les différentes régions de la cellule (fig. 2). Les particules lipidiques sont généralement de forme sphérique, elles ont un contraste moyen, souvent homogène avec parfois une zone plus claire à leur périphérie. Une enveloppe dont il est difficile de préciser la nature est présente à leur pourtour.

La comparaison, entre 1'ultrastructure des cellules sans lipides, péricytes compris, présentes chez le fœetus de porc de 69 jours au niveau de l'emplacement des tissus péricardiaque et épididymaire et l'ultrastructure des cellules sans et avec inclusions lipidiques aux mêmes niveaux des fœtus âgés de 70 jours, permet de constater que, dans les deux cas, ces cellules sont d'un même type. L'origine périvasculaire des cellules adipeuses, dont les péricytes sont un stade d'évolution, ayant été montrée dans un précédent travail sur le tissu adipeux mésentérique (VoDovar, 
DESNOYERS et FRANÇOIS, I97I), on peut conclure que l'origine des premières cellules avec des inclusions lipidiques des tissus adipeux péricardiaque et épididymaire est également périvasculaire.

\section{II. - Prolifération et évolution des préadipocytes péricardiaque et épididymaire chez le foetus de Porc jusqu'à la naissance}

Précisons dès maintenant que la prolifération des préadipocytes et l'évolution des préadipocytes sans et avec des inclusions lipidiques sont des processus qui s'effectuent sans interruption au cours de la voie foetale à partir de 1'apparition des premières cellules adipeuses. Autrement dit, il n'y a pas de stock de cellules adipeuses à partir desquelles les tissus adipeux se développeraient comme certains travaux l'ont fait croire (organe primitif de WASSERMANN, I926). D'autre part, il parait évident que la vitesse de prolifération des préadipocytes est liée à l'édification du système vasculaire et que de nombreux préadipocytes croissent surtout au stade de la formation des capillaires sanguins.

Dès que les péricytes se sont détachés de la paroi des capillaires sanguins pour devenir des cellules libres, comme cela a été démontré antérieurement (Vodovar, DeSNOYERS, FrançOIS, I97I ; DeSNOYers, I973), leurs projections cytoplasmiques diminuent tandis que le nombre des mitochondries et le réticulum endoplasmique augmente jusqu'à l'apparition des premières inclusions lipidiques. On observe généralement un ou plusieurs foyers lipidiques disséminés sans ordre apparent dans la cellule (fig. 2), leurs lipides sont homogènes avec parfois une zone plus claire ou plus foncée à la périphérie ; ces inclusions lipidiques sont entourées par une enveloppe que nous n'assimilerons pas à une cytomembrane.

Plusieurs inclusions lipidiques peuvent apparaître en même temps et très proches les unes des autres, dans ce cas elles fusionnent rapidement et après leur fusion on suit, tout au long de l'accumulation, l'évolution d'une masse lipidique centrale entourée par des inclusions de petites tailles en nombre variable qui fusionnent avec cette masse centrale (fig. 5). Lorsque les premières inclusions sont dispersées dans le cytoplasme, il se forme généralement plusieurs centres de fusionnement dont la masse lipidique s'accroît par fusion des nouvelles inclusions apparues à leur pourtour (fig. 6). Quoi qu'il en soit, le stade "adipocyte " est toujours caractérisé par la fusion de toutes les inclusions en une seule masse.

Au fur et à mesure de leur remplissage par les lipides, les préadipocytes changent progressivement de forme; le fait que l'adipocyte soit sphérique ou ovale, indépendemment de l'incidence des coupes, est conditionné par l'aspect du préadipocyte au moment de l'apparition des premières inclusions lipidiques. Les préadipocytes, dont l'extension cytoplasmique est située d'un seul côté du noyau, deviennent rapidement sphériques et leur noyau reste à la périphérie de la cellule alors que les préadipocytes, avec des extensions cytoplasmiques de part et d'autre du noyau, prennent une forme ovale avec le noyau repoussé d'un côté de la cellule, et, dans la plupart des cas, à mi-distance des deux pôles (fig. 6). Le volume des préadipocytes augmente plus ou moins rapidement en fonction de la quantité de lipides accumulés.

Le noyau, de forme généralement ovale au début de 1'accumulation des lipides, devient sphérique lorsqu'il est déplacé vers la périphérie, puis sous la poussée des lipides, prend des formes diverses telles qu'en croissant, triangulaire entre autre 
(fig. 2). L,e nucléoplasme devient moins contrasté et plus homogène et bien que cela n'ait pas été démontré, il semble généralement que le volume du noyau diminue au cours de l'accumulation des lipides. Les mitochondries parfois peu différenciées chez les jeunes préadipocytes évoluent rapidement, et, au moment de l'apparition des lipides leur matrice fondamentale, jusqu'alors peu contrastée le devient, dans la plupart des cas, davantage et leurs crêtes sont généralement régulières et plus apparentes. Au fur et à mesure que la cellule se remplit de lipides, les mitochondries, comme les autres organites cytoplasmiques sont soit repoussées à la périphérie et tassées contre la membrane plasmique (fig. 5), soit enserrées entre les inclusions lipidiques et rapidement dégradées (fig. 6). L'appareil de Golgi disparaît généralement au début de l'accumulation lipidique, tandis qu'au cours de celle-ci, on trouve du REL, en quantité variable et que le RER, tout d'abord fragmenté, disparait de façon irrégulière suivant les cellules et suivant les régions d'une même cellule (fig. 5 et 6 ). La trame cytoplasmique qui, au début de l'accumulation des lipides, relie les fractions cytoplasmiques, s'étire puis se rompt et celles-ci sont rapidement dégradées (fig. 7). Lorsque toutes les inclusions lipidiques ont fusionné en une seule masse, les cellules, sont alors considérées comme des adipocytes.

\section{III. - Évolution des adipocytes péricardiaques et épididymaires du faetus de Porc}

La présence d'adipocytes dans les tissus adipeux péricardiaques et épididymaires est observée à des temps variables après l'apparition des préadipocytes avec les premières inclusions lipidiques. A partir du moment de l'apparition des adipocytes, on observe à tous les âges du foetus, côte à côte, tous les éléments d'un tissu adipeux notamment, des préadipocytes sans inclusions lipidiques, des préadipocytes avec des inclusions variables en nombre et en volume et des adipocytes (fig. 8). Chez le foetus, malgré l'augmentation constante du nombre de cellules adipeuses, le nombre des adipocytes nous apparaît réduit par rapport au nombre de préadipocytes : cela nous semble être la conséquence de la répartititon préférentielle des lipides dans les préadipocytes sans lipides, puis dans les préadipocytes avec des inclusions lipidiques en faible quantité, ce qui entraîne une transformation lente des préadipocytes. Chez le fotus de Porc, la plupart des adipocytes ont un anneau périphérique important et des organites cellulaires encore nombreux et assez bien conservés, ce qui signifie que la maturation des adipocytes est relativement peu avancée. Enfin, quel que soit l'âge des foetus, le diamètre des adipocytes ne dépasse pas $80 \mu \mathrm{m}$.

D'une façon générale, on peut dire que si l'évolution et la maturation des cellules adipeuses des tissus adipeux péricardiaque et épididymaire sont comparables, ce dernier tissu a néanmoins un développement légèrement plus rapide.

\section{DISCUSSION}

I,es observations faites sur des coupes de tissus adipeux péricardiaques et épididymaires de fotus de Porc à différents temps de la gestation nous ont permis de mettre en évidence le moment d'apparition des premières cellules adipeuses de 
ces tissus. L'étude structurale et ultrastructurale des cellules présentes aux emplacements des tissus adipeux péricardiaque et épididymaire, c'est-à-dire les cellules disséminées autour des capillaires sanguins et les péricytes et des premiers préadipocytes avec des inclusions lipidiques nous a permis de mettre en évidence une filiation entre les cellules périvasculaires, les préadipocytes et les adipocytes dans les deux tissus étudiés.

Les observations, sur la prolifération et l'évolution des préadipocytes sans et avec inclusions lipidiques et des adipocytes pendant la période comprise entre le $70^{e}$ jour de la gestation et la naissance du porcelet, nous ont permis de constater qu'il n'existe pas d'organe primitif représenté par un stock de préadipocytes (WASSERMANN, I926), mais que la prolifération des préadipocytes est un processus continu. Nos observations, en accord avec le précédent travail (Vodovar, Desnoyers et FrançoIs, I97I), nous permettent de dire que la vitesse de prolifération des préadipocytes, et par conséquent, le développement des deux tissus adipeux étudiés, sont étroitement liés à 1'édification des capillaires sanguins, qui à ce moment libèrent un nombre important de préadipocytes.

\section{A. - Apparition et origine des tissus adipeux péricardiaques et épididymaires}

Le moment d'apparition des tissus adipeux péricardiaque et épididymaire chez le foetus de Porc, dans nos conditions d'expériences, a lieu aux environs du $70^{\mathrm{e}}$ jour de la gestation, c'est-à-dire à un stade relativement précoce de la gestation si nous le comparons au tissu adipeux mésentérique étudiée précédemment chez la même espèce (Vodovar, Desnoyers et FrançoIs, I97I), dont l'apparition se fait

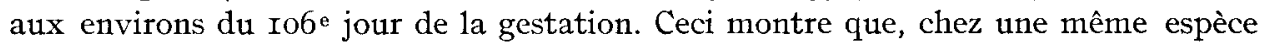
et dans les mêmes conditions d'expérience, les tissus adipeux des différents emplacements apparaissent à des temps variables. On peut présumer que pour des espèces différentes les tissus adipeux situés à des emplacements correspondants pourront avoir des moments d'apparition différents.

La variation individuelle concernant le moment d'apparition du tissu adipeux péricardiaque semble être très limitée et celle du tissu adipeux épididymaire ne dépasse pas 48 heures pour les fœtus de Porc d'une même portée ou de portées différentes. Cette faible variabilité observée pour le tissu adipeux épididymaire avait déjà été constatée pour le tissu adipeux mésentérique.

Au stade actuel de nos investigations, nous ne pouvons pas dire dans quelle mesure l'apparition et la prolifération des cellules adipeuses pourraient être décalées dans le temps si les conditions expérimentales et en particulier nutritionnelles étaient différentes. D'autre part, nous ne savons pas encore dans quelle mesure le développement des tissus adipeux du fœtus de Porc pourra influencer leur développement après la naissance.

L'observation chez le foetus de Porc de la structure et de l'ultrastructure des cellules présentes à 1'emplacement des tissus adipeux étudiés, au moment de l'apparition des premières cellules avec des inclusions lipidiques, nous a permis d'établir une filiation entre les péricytes d'origine périvasculaire, et les cellules adipeuses. Les résultats de nos investigations confirment le travail antérieur concernant le tissu adipeux mésentérique du fœtus de Porc (Vodovar, Desnovers et François, 
I97I) qui pour la première fois en microscopie électronique avait mis en évidence la filiation entre les cellules périvasculaires et les adipocytes. Nos observations concordent également avec les résultats des travaux de Simon (I963, I965) pour ce qui est de l'origine des adipocytes que l'auteur a étudiée en microscopie photonique chez le fœtus humain et l'homme adulte en se basant sur les caractères tinctoriaux des préadipocytes. Par contre, nos observations sont en désaccord avec les théories anciennes, faites en microscopie photonique (FLEMMING, I87 I et d'autres), selon lesquelles les adipocytes seraient des cellules conjonctives (fibroblastes) remplies de lipides ; elles sont également en désaccord avec les travaux en microscopie électronique de NaPolitano (I963) sur le tissu adipeux épididymaire du Rat car, si nous trouvons dans le tissu adipeux épididymaire du fœetus de Porc un certain nombre de cellules de type fibroblastiques, nous pensons que ces cellules ne deviendront jamais des adipocytes.

\section{B. - Évolution des préadipocytes péricardiaques et épididymaires}

\section{Apparition et emplacement des inclusions lipidiques.}

Contrairement à ce qui avait été observé pour le tissu adipeux mésentérique du fœus de Porc où la présence de glycogène pouvait être considérée comme annonciatrice d'une lipogénèse prochaine (Vodovar, Desnoyers et François, I97I), dans le cas des tissus adipeux péricardiaques et épididymaires, qui apparaissent beaucoup plus précocement dans l'organisme, ni cet indice ni aucun autre ne permettent de prévoir à quel moment les premières inclusions lipidiques apparaîtront dans les cellules de ces tissus.

Le glycogène, présent en abondance dans les cellules des tissus adipeux péricardiaque et épididymaire du fœetus de Porc vers la fin de la gestation, n'a été que très exceptionnellement observé à des stades précoces de la formation de ces deux tissus. On peut donc penser, sauf si le glycogène pour une raison indéterminée n'a pas été mis en évidence, qu'au moment de l'apparition de ces tissus et pendant un certain temps après cette apparition, les lipides accumulés dans les cellules adipeuses des tissus adipeux péricardiaque et épididymaire sont surtout apportés par le sang maternel et que la lipogénèse intracellulaire ne devient importante qu'une semaine environ avant la naissance, ce qui coïncide avec l'apparition des premières cellules adipeuses du tissu adipeux mésentérique (VodOvar, DESNOYERS et FrançOIS, I97I).

En ce qui concerne l'augmentation de la population mitochondriale et le développement du RER dans la cellule que nous avons constatés au moment de l'apparition des premières inclusions lipidiques, ces observations concordent avec les travaux de NAPOLITANO (I963) bien que celui-ci ait travaillé sur le tissu adipeux épididymaire d'une autre espèce, le Rat, et environ 7 jours après la naissance.

\section{Nature des premières cellules adipeuses péricardiaques et épididymaires.}

Comme on le sait les tissus adipeux péricardiaque et épididymaire sont des tissus adipeux à graisse blanche. Cependant, dans la littérature, des opinions contradictoires ont été émises quant à la nature des premières cellules adipeuses des différents emplacements. NAPOLITANo et FAWCETT (I958), après leurs travaux sur la Souris et le Rat, assurent que jamais le tissu adipeux à graisse brune ne devient du 
tissu adipeux à graisse blanche alors que SImoN (I962-I965) affirme que pour toutes les espèces et à tous les emplacements, les tissus adipeux ont pour origine des cellules à graisse brune qui selon les espèces et les emplacements, restent sans changement ou se transforment rapidement en cellules à graisse blanche. Les travaux de DAwkins et HUL, (I964) sur le I,apin, et de GEMmEr, et al. (I972) sur l'Agneau viennent corroborer l'opinion de Simon.

Nos observations sur les tissus adipeux péricardiaque et épididymaire du fœtus de Porc nous permettent de confirmer que, comme pour le tissu adipeux mésentérique de cette même espèce, les cellules adipeuses de ces deux tissus sont à tous les moments des cellules à graisse blanche caractérisées aussi bien par l'aspect de leurs lipides que par celui de leurs éléments cellulaires, en particulier par les mitochondries peu nombreuses au stade initial et n'ayant pas d'inclusions opaques dans leur matrice.

Ces observations tendent à confirmer les affirmations de NAPOLITANo et FAWCETT (I958), toutefois, au stade actuel de nos investigations, nous ne pouvons pas dire si au niveau des tissus adipeux des autres emplacements et en particulier pour les tissus adipeux à graisse blanche voisins des tissus adipeux à graisse brune, cette constatation se révélerait également valable.

\section{3. Évolution des cellules pendant l'accumulation des lipides.}

L'évolution de la forme des cellules adipeuses, qui tendent généralement vers la sphère, dépend, pour une large part, de la morphologie cellulaire au moment de 1'apparition des premières inclusions lipidiques, de la répartition des inclusions dans les cellules et de l'emplacement des cellules par rapport aux éléments qui les entourent.

Chez le fœtus de Porc, les cellules adipeuses péricardiaque et épididymaire ne sont pas en contact les unes avec les autres pendant un mois environ après leur apparition, ce qui permet d'étudier assez facilement les modifications de leur forme sous la seule action de l'accumulation des lipides.

Les observations effectuées permettent de constater, en accord avec ce que nous avions vu pour le tissu adipeux mésentérique (VODOVAR, Desnoykrs et FrançoIs, I97I) que l'accumulation lipidique à ces deux emplacements est lente, du fait de l'apparition précoce des tissus, ce qui facilite l'étude de l'évolution des organites intracellulaires.

En conclusion nous pouvons dire :

- que l'apparition des premiers éléments des tissus adipeux péricardiaque et épididymaire se fait, dans nos conditions d'expérience, aux environs du $70^{\mathrm{e}}$ jour de la gestation soit 45 jours avant la naissance ;

- que l'origine des adipocytes de ces deux tissus est périvasculaire et que l'intensité de la prolifération des préadipocytes est étroitement liée à la formation des capillaires sanguins;

- que la prolifération des préadipocytes et leur évolution, jusqu'au stade adipocyte, est un processus continu et qu'il n'existe pas de tissu primitif à partir duquel les tissus adipeux se développeraient ;

- que l'évolution des tissus adipeux péricardiaque et épididymaire est assez lente au stade périnatal chez l'espèce étudiée. 


\section{REMERCIEMENTS}

Nous remercions M. J. Fevre pour la mise à notre disposition des foetus de porcs. Le tirage des photographies a été effectué par M. G. PaIllard. Ce travail a été effectué avec l'aide financière de 1'I. N. S. E. R. M. (ATP no r9) et de l'I. N. R. A. (ATP Tissu adipeux).

\section{SUMMARY}

\section{APPEARANCE, ORIGIN AND DEVELOPMENT OF EPIDIDYMAI. AND PERICARDIAC ADIPOSE TISSUES IN PIG FOETUS}

The time of appearance, origin and development of peridardiac and epididymal adipose tissues are studied in pig foetuses varying in age between 60 and $I_{1} 3$ days (day before presumed birth). In our experimental conditions, appearance of the first units of pericardiac and epididymal adipose tissues occurs around day $7^{\circ}$ of pregnancy.

The ultrastructural study of lipid-free cells (pericytes included) and primor cells with lipid inclusions present in the pericardiac and epididymal adipose tissues led us to conclude a perivascular origin for these two adipose tissues.

The appearance of free endothelial cells, the preadipocytes, is shown to be a continuous process all during foetal life, and the intensity of proliferation of the future adipocytes closely related to the formation of new blood capillaries.

Taking into account the ultrastructural aspect of cells when the first lipid inclusions appear, and their development, it can be confirmed at any moment that these cells are adipose cells of white fat tissue.

The results are discussed and, as no data are available on these adipose tissues, they are compared with existing results on adipose tissues in other sites.

\section{RÉFÉRENCES BIBI,IOGRAPHIQUES}

Barrnett R. J., I962. In : Adipose tissue as an organ. Thomas Ch. C., Springfield, Illinois, Kinsell L. W. Ed., 3-78.

DAwkins M. J., HULL D., I964. Brown adipose tissue and the response of new-born rabbits to cold. J. Physiol. (London), 172, 2 I6.

DESNOYeRs F., 1973. Étude morphologique des adipocytes et leur évolution chez le Porc et chez le Rat. Thèse de Doctorat, Paris.

Flemming W., I87x. On the formation and regression of fat cells in connective tissue with comment on the structure of the latter. Arch. R. mikr. Anat., 7, 32-47.

Gemmell R. T., Bell A. W., Alexander G., 1972. Morphology of adipose cells in lambs at birth and during subsequent transition of brown to white adipose tissue in cold and in warm conditions. Am. J. Anat., 133, 143.

LeEson C. R., LeESON T. S., r97o. Staining methods for sections of epon embedded tissues for light microscopy. Can.J. Zool., 48, I89-rgo.

LuFt J., rg6r. Improvments in epoxy resin embedding methods. J. biophys. biochim. Cytol., 9, $409-4 \mathrm{I} 4$.

Millonig G., I96r. The advantages of a phosphate buffer for OsO4 solutions in fixation. J.appl. Physics, 32, I637.

Napolitano L., I963. The differentiation of white adipose cells : an electron microscopic study. $J$. Cell. Biol., 18, 663-679.

Napolitano L., Fawcett D. W., I958. The fine structure of brown adipose tissue in the newborn mouse and Rat. J. Biophys. Biochem. Cytol., 4, 685-703.

REYNoldS E., I963. The use of lead citrate at high $\mathrm{pH}$ as an electron opaque strain in electron microscopy. J. Cell. Biol., 17, 208-212. 
Sheldon H., I965. Morphology of adipose tissue : a microscopic anatomy of fat. In : Handbook of Physiology : adipose tissue, 5, r25. Renold A. E., Cahill G. F. Jr., Am. Phys. Soc. Washington D. C.

Srmov G., I962. Genèse et structure du tissu adipeux chez l'Homme. Acta anat., 48, 232-24r.

Simon G., I965. Histogenesis. In: Handbook of physiology : adipose tissue, 5, ror-Io7. Renold A. E., CaHill G. F. Jr., Am. Phys. Soc. Washington D. C.

Told C., I87o. Contribution to the histology and physiology of adipose tissue. Sitzber. Akad. Wiss. Wien. Math. Naturwiss., 62, 445-452.

Vodovar N., Desnoyers F., Françors C., r97r. Origine et évolution des adipocytes mésentériques du porcelet avant la naissance. Aspect ultrastructural. J. Micr., 11, 265-284.

Wassermane F., I926. The fat organs of man : development, structure and systematic place of socalled adipose tissue. Z. Zellforsch. Mikroskop. Anat. Abt. Histochimie, 3, 325-328.

Wells H. G., I940. Adipose tissue - a neglected subject. J.A. M. A., 114, 2 I77-2I83.

\section{ABRÉVIATIONS UTILISÉES DANS LES PLANCHES}
A : Adipocyte
B : Lame basale
C : Capillaire sanguin

CA : Preadipocyte avec des inclusions lipidiques

$\mathrm{G}$ : Appareil de Golgi

L : Lipides

M : Mitochondries

$\mathrm{N}$ : Noyau

$\mathrm{P}$ : Péricyte

PR : Preadipocyte sans inclusion lipidique

TC : Travée cytoplasmique 


\section{PLANCHE I}

\section{FIG. I}

Coupe de péricarde fibreux prélevé chez un fœtus de Porc au $69^{\text {e }}$ jour de la gestation. Les cellules, de même type, n'ont pas d'inclusions lipidiques et elles sont présumées être de futurs cellules adipeuses. Suivant l'incidence de la coupe, la forme de la section de ces cellules, PR, est soit allongée, soit circulaire $(\times 5500)$.

\section{FIG. 2}

Coupe de péricarde fibreux prélevé, à un niveau correspondant à celui de la figure $I$, sur un fotus de 70 jours. On observe les premières cellules avec des inclusions lipidiques CA. Noyau $\mathrm{N}$, Lipides $L$, cellules sans inclusion lipidique PR $(\times 4000)$. 


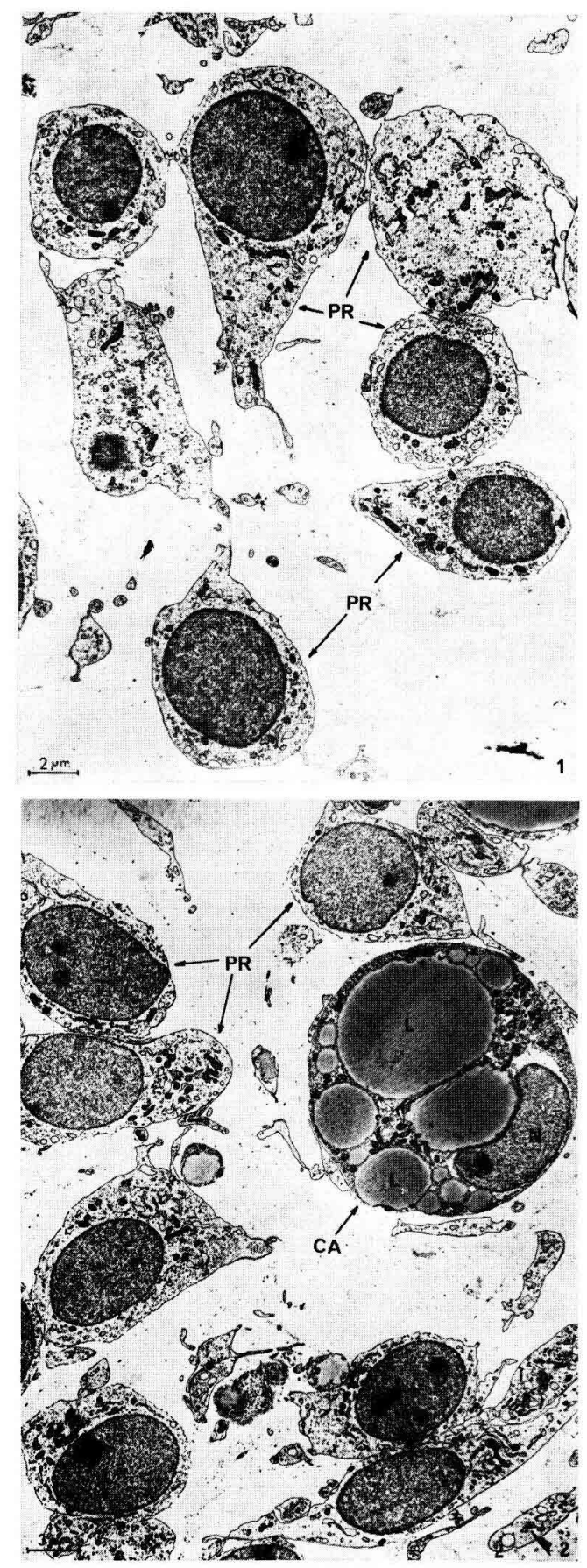

FrancoIse DFSNOYERS, N. VODOVAR 


\title{
PLANCHE II
}

\author{
Fig. 3
}

Coupe à l'emplacement du tissu adipeux épididymaire d'un fœetus de Porc au $69^{\mathrm{e}}$ jour de la gestation. On observe à côté de trois cellules sans inclusion lipidique PR un capillaire sanguin $C$ entouré d'une lame basale B. $(\times 5.300)$.

FIG. 4

Coupe de péricarde fibreux d'un fotus de 69 jours au niveau d'un capillaire sanguin, C, ouvert entouré d'un péricyte, $P$, et de deux cellules, $P R$, présumées être de futurs adipocytes. Lame basale, B ; Appareil de Golgi, G ; Noyau, N. $(\times 5.500)$. 

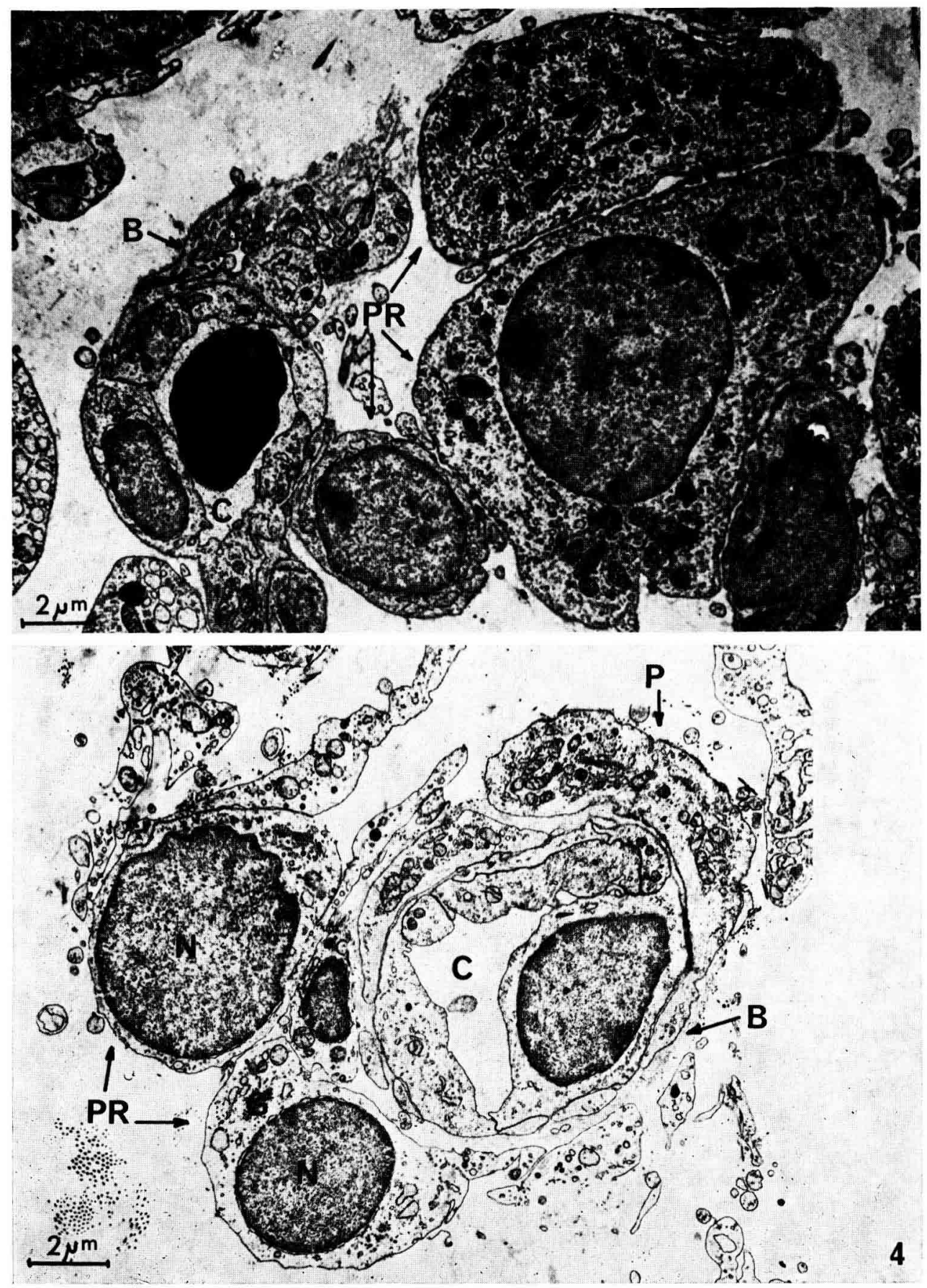

Frangoise DESNOYERS, N. VODGVAR 


\title{
PLANCHE III
}

\author{
Fig. 5
}

Coupe d'une cellule adipeuse CA de forme ovale présentant pratiquement un seul foyer lipidique principal L. Le noyau $\mathrm{N}$ est repoussé à un pôle de la cellule. Les mitochondries $M$ sont rares. $(\times 7.000)$.

FIG. 6

Coupe d'une cellule adipeuse CA de forme semblale à celle de la figure 7 mais avec deux foyers lipidiques principaux $L$ situés de part et d'autre du noyau $\mathbf{N}$ qui est repoussé latéralement. $(\times 7.000)$. 


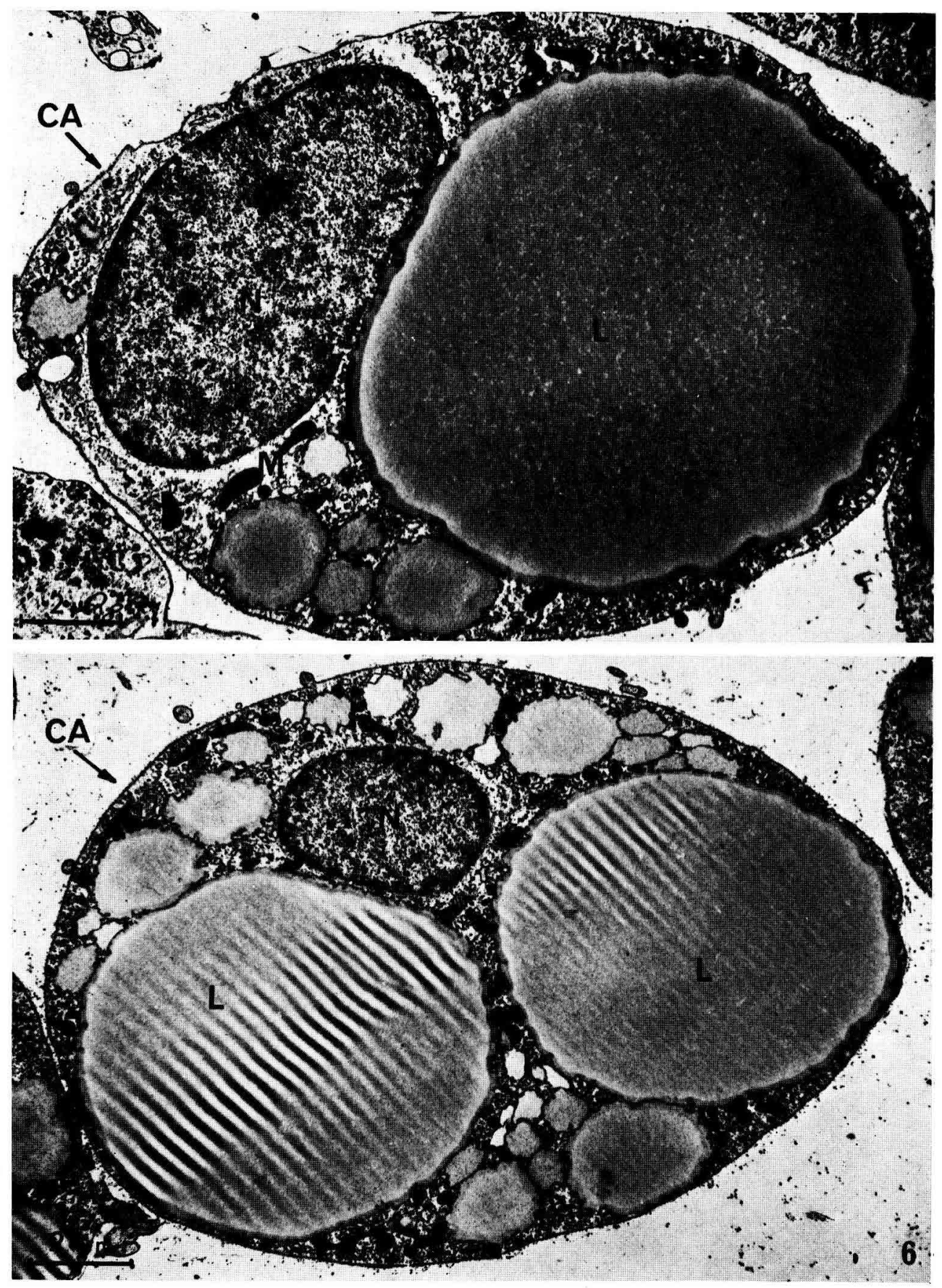

FrançoIse DESNOYERS, N. VODOVAR 


\section{PLANCHE IV}

\section{FIG. 7}

Coupe d'une cellule adipeuse CA dans laquelle on observe des travées cytoplasmiques TC en cours de dégradation et des éléments cellulaires enserrés entre les masses lipidiques $L$. $(\times 3.500)$. 


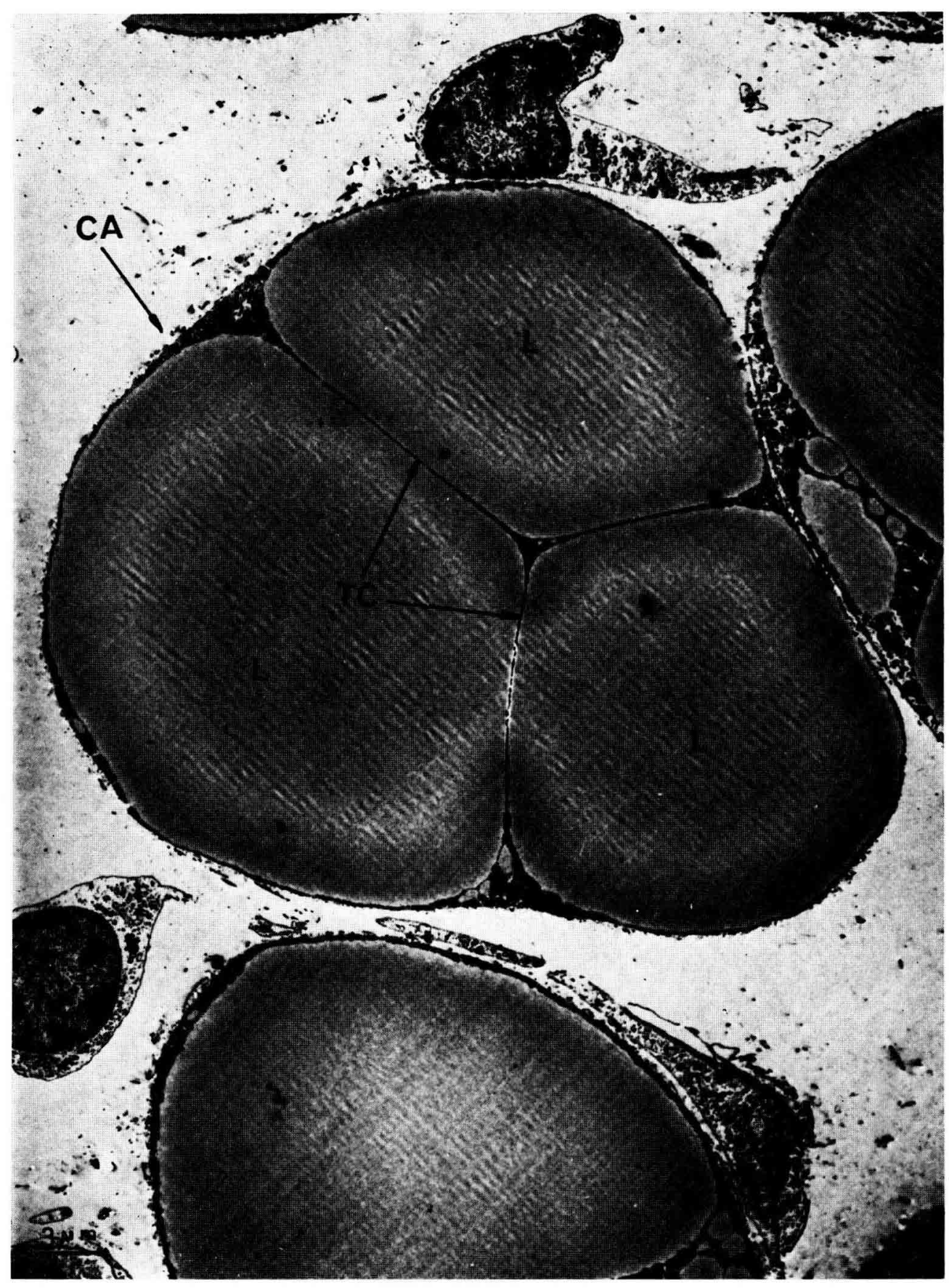


PLANCHE V

Fig. 8

Coupe d'un tissu adipeux permettant d'observer côte à côte des préadipocytes sans inclusion lipidique $P R$, des préadipocytes avec des inclusions lipidiques $\mathrm{CA}$, et des adipocytes $\mathrm{A}$. Capillaire sanguin, C. $(\times 3.500)$. 


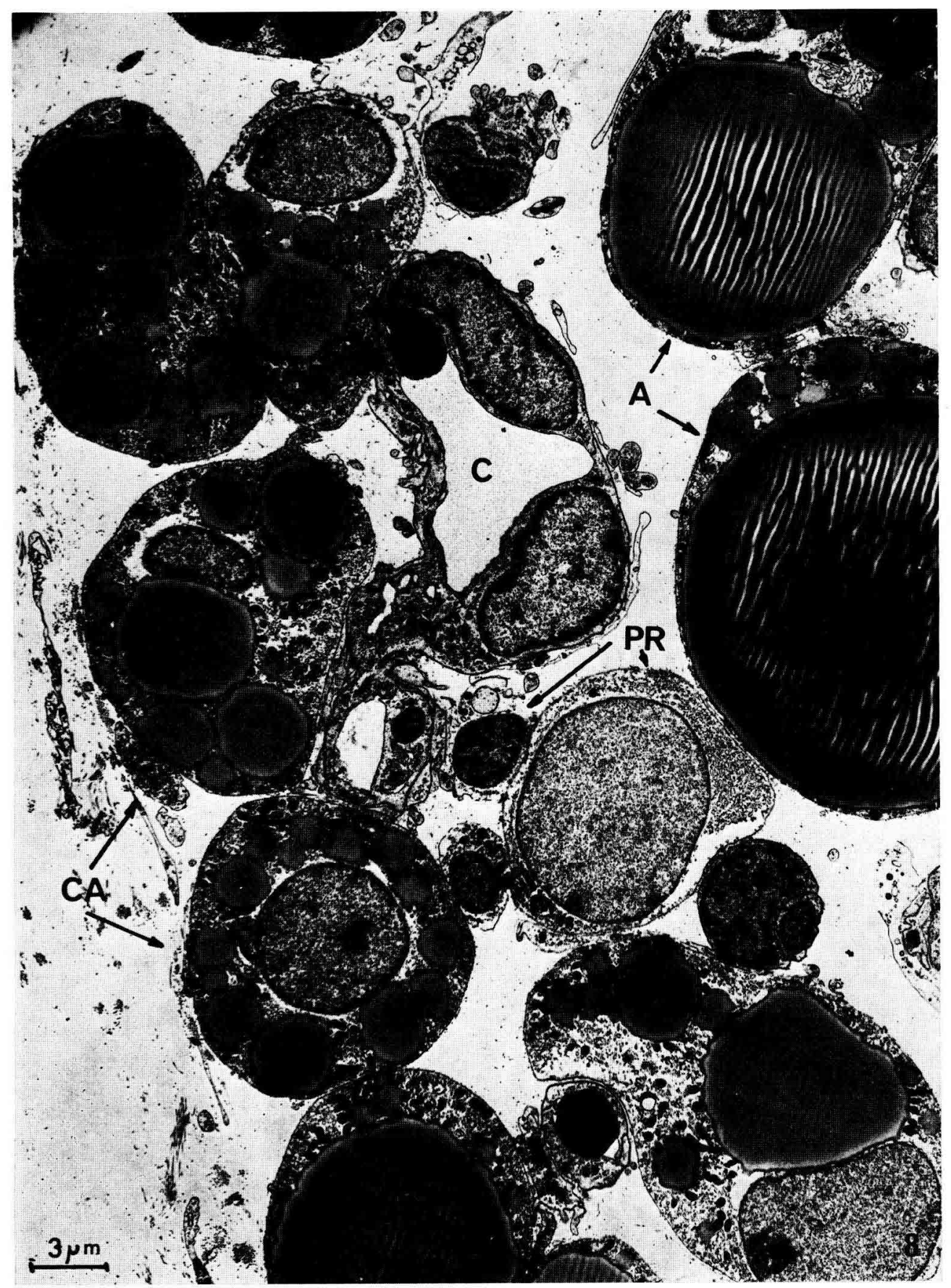

Francoise DESNOYERS, N. VODOVAR 